\title{
Antibody-Drug Gold Nanoantennas with Raman Spectroscopic Fingerprints for in Vivo Tumour Theranostics
}

\author{
Joao Conde \\ Massachusetts Institute of Technology \\ Chenchen Bao \\ Institute of Micro and Nano Science and T, P.R. China \\ Daxiang Cui \\ Institute of Micro and Nano Science and T, P.R. China
}

See next page for additional authors

Follow this and additional works at: https://arrow.tudublin.ie/nanolart

Part of the Physical Sciences and Mathematics Commons

\section{Recommended Citation}

Conde, J. et al. (2014) Journal of controlled release, 2014 Jun 10;183:87-93. doi:10.1016/

j.jconrel.2014.03.045

This Article is brought to you for free and open access by the NanoLab at ARROW@TU Dublin. It has been accepted for inclusion in Articles by an authorized administrator of ARROW@TU Dublin. For more information, please contact arrow.admin@tudublin.ie, aisling.coyne@tudublin.ie,gerard.connolly@tudublin.ie.

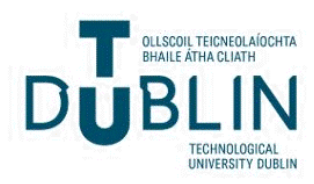




\section{Authors}

Joao Conde, Chenchen Bao, Daxiang Cui, Pedro Baptista, and Furong Tian

This article is available at ARROW@TU Dublin: https://arrow.tudublin.ie/nanolart/58 


\section{Antibody-Drug gold nanoantennas with Raman spectroscopic fingerprints for in vivo tumour theranostics}

João Conde ${ }^{a, b, c, *}$, Chenchen Bao ${ }^{d}$, Daxiang Cui ${ }^{d}$, Pedro V. Baptista ${ }^{b}$ and Furong Tian $^{e, *}$

a Instituto de Nanociencia de Aragon (INA), Universidad de Zaragoza, Zaragoza, 50018, Spain.

${ }^{\mathrm{b}}$ CIGMH, Departamento de Ciências da Vida, Faculdade de Ciências e Tecnologia, Universidade Nova de Lisboa, Campus de Caparica, 2829-516 Caparica, Portugal.

${ }^{\mathrm{c}}$ Current address: Massachusetts Institute of Technology, Harvard-MIT Biomedical Engineering Center, E25-449, Cambridge, Massachusetts, USA.

${ }^{\mathrm{d}}$ Department of Bio-Nano Science and Engineering, National Key Laboratory of Micro/Nano Fabrication Technology,Institute of Micro\&Nano Science and Technology, P.R.China.

${ }^{\mathrm{e}}$ Focas Research Institute, Dublin Institute of Technology, Camden Row, Dublin, Ireland.

[*] Corresponding Authors

Dr. Furong Tian

Focas Research Institute, Dublin Institute of Technology, Ireland.

E-mail: furong.tian@dit.ie

Dr. João Conde

Massachusetts Institute of Technology, Harvard-MIT Biomedical Engineering Center, USA. E-mail: jdconde@mit.edu

Keywords: Nanotheranostics; Antibody-Drug conjugates; Raman fingerprints; SERS; in vivo tumour targeting.

\section{ABSTRACT}

Inspired by the ability of SERS nanoantennas to provide an integrated platform to enhance disease targeting in vivo, we developed a highly sensitive probe for in vivo tumoral recognition with the capacity to target specific cancer biomarkers such as epidermal growth factor receptors (EGFR) on human cancer cells and xenograft tumour models. Here, we used $\sim 90 \mathrm{~nm}$ gold nanoparticles capped by a Raman reporter, encapsulated and entrapped by larger polymers and a FDA antibody-drug conjugate - 
Cetuximab (Erbitux®) - that specifically targets EGFR and turns off a main signalling cascade for cancer cells to proliferate and survive. These drug/SERS gold nanoantennas present a high Raman signal both in cancer cells and in mice bearing xenograft tumours. Moreover, the Raman detection signal is accomplished simultaneously by extensive tumour growth inhibition in mice, making these gold nanoantennas ideal for cancer nanotheranostics, i.e. tumour detection and tumoral cell inhibition at the same time.

\section{INTRODUCTION}

Surface-enhanced Raman Spectroscopy (SERS) has led the way in terms of use of spectroscopic methods for signal enhancement by nanostructured metal surfaces towards in vivo tracking of biomolecules trafficking.[1-3]

When light interacts with a molecule, it can be absorbed, transmitted, or scattered, which may be a consequence from an elastic (Rayleigh scattering) or inelastic (Raman scattering)

collision. Raman spectroscopy relies on the modification of frequency of light when it is inelastically scattered by molecules or atoms resulting in fingerprint information on molecular structure or environment.[4] SERS has been extensively used for molecular/ion detection and bioimaging applications since it minimizes photoblinking or photobleaching from conventional fluorophores, decreases signal-to-noise ratio in vitro and in vivo, as usually Raman reporters are stable and with large quantum yields.[5-8] Colloidal metal nanoparticles (especially silver and gold) act as good SERS enhancers, via surface electromagnetic field enhancement and chemical contribution.[9] Gold nanoparticles (AuNPs) covered by Raman reporters have been used for SERS to detect cancer cells in vitro and tumours in vivo.[10-13] Raman reporters combined with AuNPs produce an optical contrast to distinguish between cancer and normal cells and their conjugation with antibodies allowed them to determine the expression of relevant biomarkers in molecular imaging.[13] Qian et al. reported that, when conjugated to tumour targeting ligands, these SERS-NPs were able to target tumour markers 
at surface of malignant cells, such as epidermal growth factor receptor (EGFR) and used to locate tumours in cancer models.[14] Nevertheless, using SERS-NPs as a nanotheranostic tool to detect xenograft tumours and the same time inhibit tumour size and progression has never been reported before.

Here, we used 90 nm AuNPs covered by DTTC (3,3'-Diethylthiatricarbocyaniniodid) Raman reporters, that were encapsulated and entrapped by larger polymers (i.e. $5000 \mathrm{Da}$ thiol- and $\mathrm{COOH}$-modified polyethylene glycol coat). These polymers served as substrates for the binding of a FDA approved antibody-drug conjugate, the Cetuximab (Erbitux®, Merck).

Cetuximab is a monoclonal antibody that specifically targets EGFR, a receptor that often appears in high amounts on the surface of cancer cells and gives a signal that causes those cells to divide uncontrollably. Cetuximab binds to such receptors and turns off that signal [15] (see Figure 1).
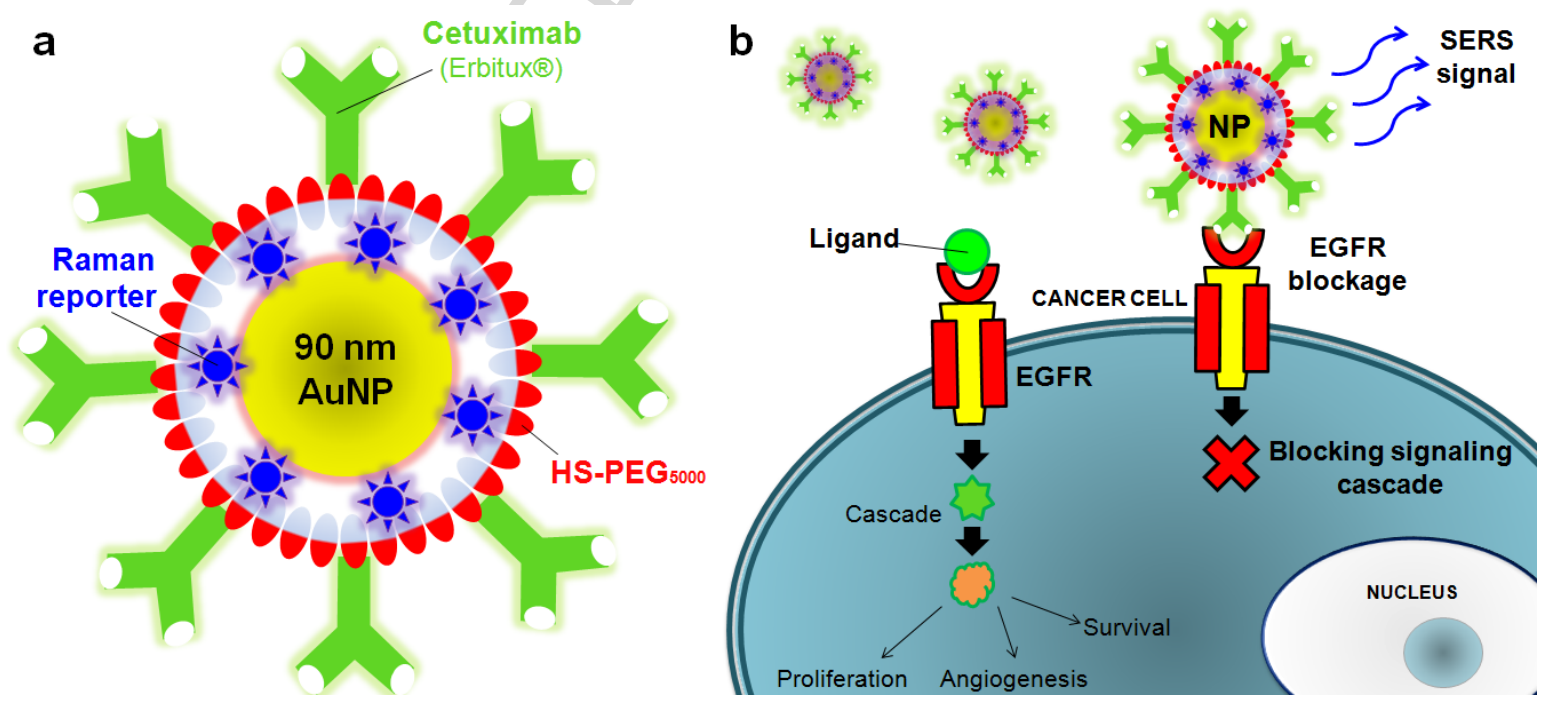

Figure 1. (A) Antibody-Drug gold SERS nanoantennas - 90nm AuNPs surrounded by a Raman reporter, encapsulated and entrapped by a larger polyethylene glycol (PEG) polymer and covered with a FDA approved antibody-drug conjugate, the Cetuximab (Erbitux $\left.{ }^{\circledR}\right)$. (B) The Drug-Raman NPs can easily bind to EGF receptors (EGFR), blocking the EGF protein from reaching the cancer cells and inhibit the signalling cascade. The activation of this cascade will consequently stop proliferation and survival of targeted cells. 
The cyanine derivative DTTC has got a huge potential application in Raman imaging, singlecell detection, cancer diagnosis, and SERS, once its emission and absorption peaks are located in the NIR region.[14;16] Our drug/SERS nanoantennas present a high Raman signal both in in vitro cancer cells and in in vivo mice bearing xenograft tumours, when compared to the low performance of DTTC as sensitive probes for in vivo detection, due to a modest Raman intensity. Moreover, extensive tumour growth inhibition in mice can be simultaneously observed through the evaluation of Raman signal.

\section{RESULTS AND DISCUSSION}

Spherical naked AuNPs with an average diameter of $91.4 \pm 8.9 \mathrm{~nm}$ confirmed by transmission electron microscopy (TEM) (Figure 2A,B) were incubated with freshly prepared DTTC reporter solution $($ Emission $=780-800 \mathrm{~nm}$ ) by adding it dropwise to a mixture of NPs and sodium dodecyl sulphate (SDS). Afterwards, a $\alpha$-Mercapto- $\omega$-carboxy polyethylene glycol (PEG) solution (HS-C ${ }_{2} \mathrm{H}_{4}$-CONH-PEG-O- $\mathrm{C}_{3} \mathrm{H}_{6}-\mathrm{COOH}$, MW. 5000Da) was added in 10-fold excess to the Raman-AuNPs, in order to fully cover the DTTC-NPs and to stabilize the gold conjugates against aggregation/clustering and precipitation, whilst increasing biocompatibility, grant chemical functionality and avoid opsonization. Actually, PEGylated AuNPs exhibit outstanding in vivo biodistribution and pharmacokinetic properties upon local or systemic administration.[17] The PEG spacer also provides the anchoring moiety for the covalent binding of amine-containing antibody-drug through carbodiimide chemistry, as previously described.[18;19] After PEG functionalization, nanoconjugates were washed in order to remove PEG excess and surfactant. Cetuximab was bound to the SERS-NPs through a carbodiimide chemistry, using an EDC/NHS coupling reaction between the carboxylated PEG spacer and the amine terminal group of the antibody (376.1 \pm 9.5 Cetuximab antibodies per nanoparticle, see Fig. S1 in Supporting Information for quantification by the Bradford assay as previously described [20]). 
Also shown are the optical extinction spectra of AuNP-conjugates with a surface plasmon resonance (SPR) band centred at $560 \mathrm{~nm}$ (Figure 2C), Raman spectra (Figure 2D), hydrodynamic size and zeta-potential data (Figure 2E) and dark-field light-scattering microscopy (Figure 2F). The gold plasmonic resonance spectra remained almost unchanged (with a shift in SPR 1-2 nm, for NPs-PEG only, NPs-DTTC-PEG [Raman NPs] and NPsDTTC-PEG-Cetux [Drug-Raman NPs]), however with a shoulder in extinction spectra around $775 \mathrm{~nm}$, characteristic of the Raman reporter, DTTC. The typical Raman spectrum acquired from the Raman reporter (DTTC dye) only, Raman-NPs and Drug-Raman NPs are depicted in Figure 2D. Raman-NPs and Drug-Raman NPs show a characteristic SERS peak at $508 \mathrm{~cm}^{-1}$. SERS spectra were acquired in solution. The consistent SERS signals from the DTTClabelled nanoparticles allow us to use the Raman spectrum as a spectroscopic fingerprint to monitor the presence of the specific Drug-Raman NPs both in in vitro and in in vivo applications. The particles' hydrodynamic diameter increased by approximately $20 \mathrm{~nm}$ after PEGylation. A considerable increase in diameter is observed upon cetuximab binding (increased $\sim 45 \mathrm{~nm}$ ) as measured by hydrodynamic light scattering (DLS) in water (Figure 2E and Fig. S2 in Supporting Information for diameter distribution histograms). The size distribution generated by DLS is based on intensity distributions. These data corroborate previous studies showing that AuNPs with a core size between 60 and $90 \mathrm{~nm}$ have high SERS efficiency in the NIR region,[21] the effective physiologic window for in vivo therapeutic applications, as blood components and water absorption is negligible.[22] Zeta-Potential data show that all nanoparticle conjugates are considered anionic with zeta potentials between -20 to $-30 \mathrm{mV}$ (Figure 2E). Figure $2 \mathbf{F}$ shows optical dark-field light scattering microscopy image of Drug-Raman NPs. As a result of the SPR properties, the $90 \mathrm{~nm}$ nanoparticles absorb and scatter light so intensely that single NPs are easily observed by the naked eye using dark-field (optical scattering) microscopy.[23] 

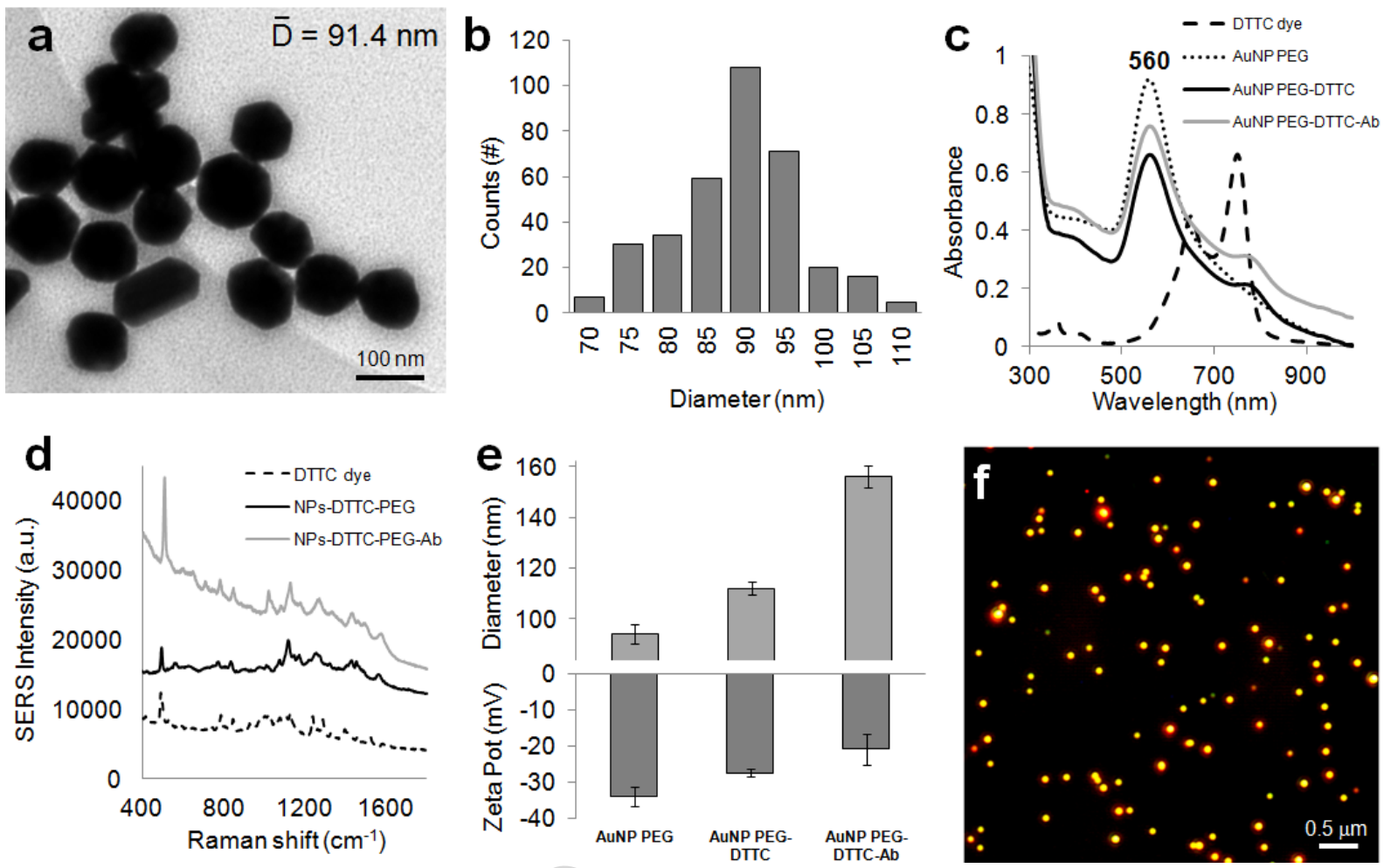

Figure 2. Physicochemical characterization of tested AuNPs (A) Transmission electron microscopy images of spherical naked AuNPs (scale bar, $100 \mathrm{~nm}$ ) with an average diameter of $91.4 \pm 8.9 \mathrm{~nm}(\mathbf{B})$. (C) Optical extinction spectra with an SPR band centred at $560 \mathrm{~nm}$. Also shown is a DTTC absorbance spectrum with characteristic peaks at 650 and $750 \mathrm{~nm}(\mathbf{D})$ Raman spectra of AuNP-conjugates with a characteristic SERS peak at $508 \mathrm{~cm}^{-1}$. (E) Hydrodynamic light scattering (DLS) in water and zeta-potential data of all AuNP-conjugates. (F) Dark-field light-scattering microscopy of Drug-Raman NPs, i.e. NPs-PEG-DTTC-Ab (scale bar, $0.5 \mu \mathrm{m}$ ).

We next investigated the capacity of SERS-coded antibody/drug AuNP conjugates to provide spectroscopic signatures in in vitro HT-adenocarcinoma cells from colorectal cancer that overexpress EGFR.[24] Cells were incubated for 24 hours with Drug-Raman NPs $\left(1.78 \times 10^{9}\right.$ NPs) and confocal microscopy images were captured in order to measure lysosome signal, using LysoTracker Green (Figure 3A) and DTTC emission signal (Figure 3B). The EGFR receptor is one of the key membrane proteins located on the cell surface and for this reason it is expected that the Drug-Raman NPs would be mostly disseminated in the cell membrane.

This is clearly seen in Figure 3B where the DTTC signal is located mainly in cell membrane and cell membrane associated contents. Some co-localization was detected as Drug-Raman 
NPs selectively accumulated in lysosomes of HT-adenocarcinoma cells (Figure 3C and Fig.

S3 in the Supporting Information). Upon endocytosis the Drug-Raman NPs usually go to the lysosomal compartments, facing a strong acidic and enzymatic environment, compromising the stability and fluorescence of the drug-Raman-AuNP conjugates.[25;26] Nevertheless, the photostability of Drug-Raman NPs associated with lysosomes was detectable and high, at least after 24 hours of cell incubation. A strong fluorescence signal from Raman reporter was also detected and distributed in all cytoplasm (Figure 3C). Figure 3D shows characteristic and strong Raman spectrum peaks of Drug-Raman NPs in HT-adenocarcinoma cells. Cell survival rates were evaluated via a standard MTT assay (Fig. S4 in Supporting Information). As expected, cell viability was inhibited for drug-Raman-AuNP only, once Cetuximab is involved in the inhibition of cancer cell proliferation and survival.
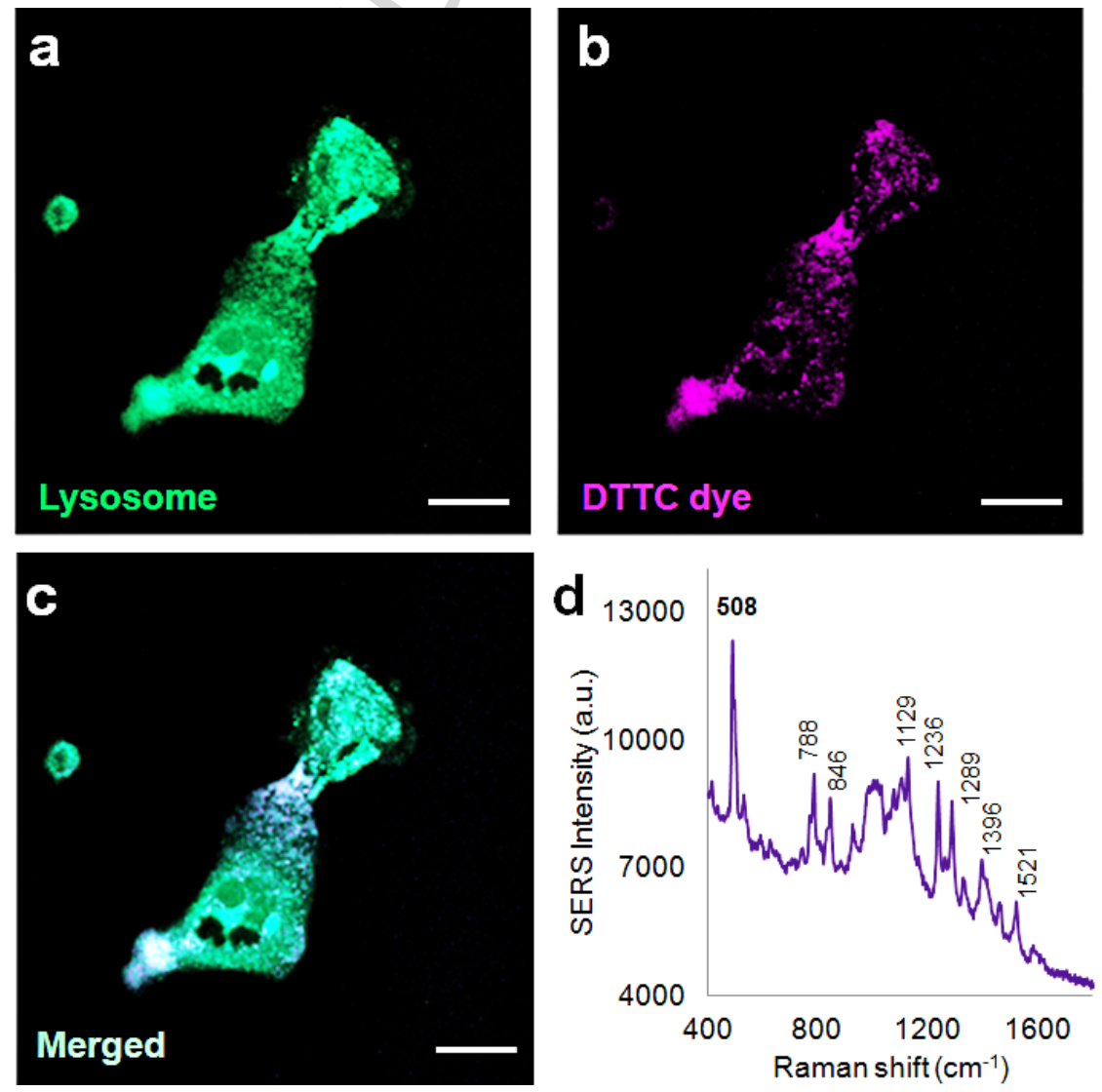

Figure 3. Representative confocal images of HT-adenocarcinoma cells incubated for 24 hours with Drug-Raman NPs $\left(1.78 \times 10^{9} \mathrm{NPs}\right)$. Shown is lysosome signal via LysoTracker Green (A), Raman reporter (DTTC dye) emission signal (B) and co-localization signal between DrugRaman NPs and lysosomes (C) (scale bars, $10 \mu \mathrm{m}$ ). (D) Characteristic Raman spectra and 
peaks of Drug-Raman NPs in HT-adenocarcinoma cells, with a characteristic SERS peak at $508 \mathrm{~cm}^{-1}$.

In order to test tumour size and nanoparticle uptake and biodistribution in whole body mice and predominantly in xenograft tumour tissue, a xenograft tumour mice model was developed. Briefly, B6 albino female mice were injected in the right leg with HT-29-luc-D6 Bioware® Cell Line (P/N 119264, PerkinElmer®), which is a light producing cell line derived from HTadenocarcinoma cells by stable transfection of the North American Firefly Luciferase gene expressed from the CMV promoter. All AuNPs conjugates were injected in mice's tails $\left(1.78 \times 10^{9} \mathrm{NPs}\right.$ per injection, $\left.50 \mu \mathrm{L}\right)$. Xenograft tumour induction was done during 8 weeks before NPs injection. Bioluminescence/Epi-fluorescence live images of mice bearing xenografts were done 72 hours after NPs systemic administration through tail vein injection. Representative tomographic images of individual mouse from each treated group $(n=10$ animals) is shown, with the same scale photon flux indicating luciferase activity (Figure 4A,B). Shown is luminescence of mice bearing xenografts treated with $1.78 \times 10^{9} \mathrm{NPs}$ of Drug-Raman NPs and Raman NPs (without antibody-drug conjugate). Excised organs from mice were also imaged for luciferase activity at $72 \mathrm{~h}$ post-tail-injection (Figure $4 \mathrm{C}$ ). These tomography images clearly depict that Drug-Raman NPs have an increased targeting xenograft tumour cells when compared to Raman NPs group (without antibody-drug conjugate), resulting in extensive $(\sim 70 \%)$ tumour size reduction as depicted by the decrease in luminescence signal in mice's leg.

Figure 4D-F shows Hematoxylin and Eosin staining (H\&E) in mice xenograft tumour tissues which confirm data from live imaging. Hematoxylin has a deep blue-purple colour, staining nucleic acids and eosin is pink and stains proteins. In a characteristic tissue, nuclei are stained blue and the cytoplasm and extracellular matrix are stained pink. As expected, sham treated group (mice without any nanoparticle's treatment) cancer cells are migrated and distributed in 
tumour tissue (Figure 4D). Severe cancer cells infiltration is also noted in Raman NPs treated group (Figure 4E). In the Drug-Raman NPs treated group there was a significant decrease in the incidence and severity of tumour clones in the whole tumour tissue, revealing the capacity of Drug-Raman NPs in reducing tumour cells in a xenograft tumour in mice (Figure 4F). Almost no cancer clones can be observed at the recovered tumour area treated with DrugRaman NPs. A large area of dead cancer cells without nuclei can be clearly seen in drugRaman NP-treated tumours (Figure 4F). Additional tumour tissue slides for sham, Raman NP and drug-Raman NP groups can be found in Fig. S5 in the Supporting Information. No toxicity or other physiological complications were observed for the animals after 1 month of NPs injection.
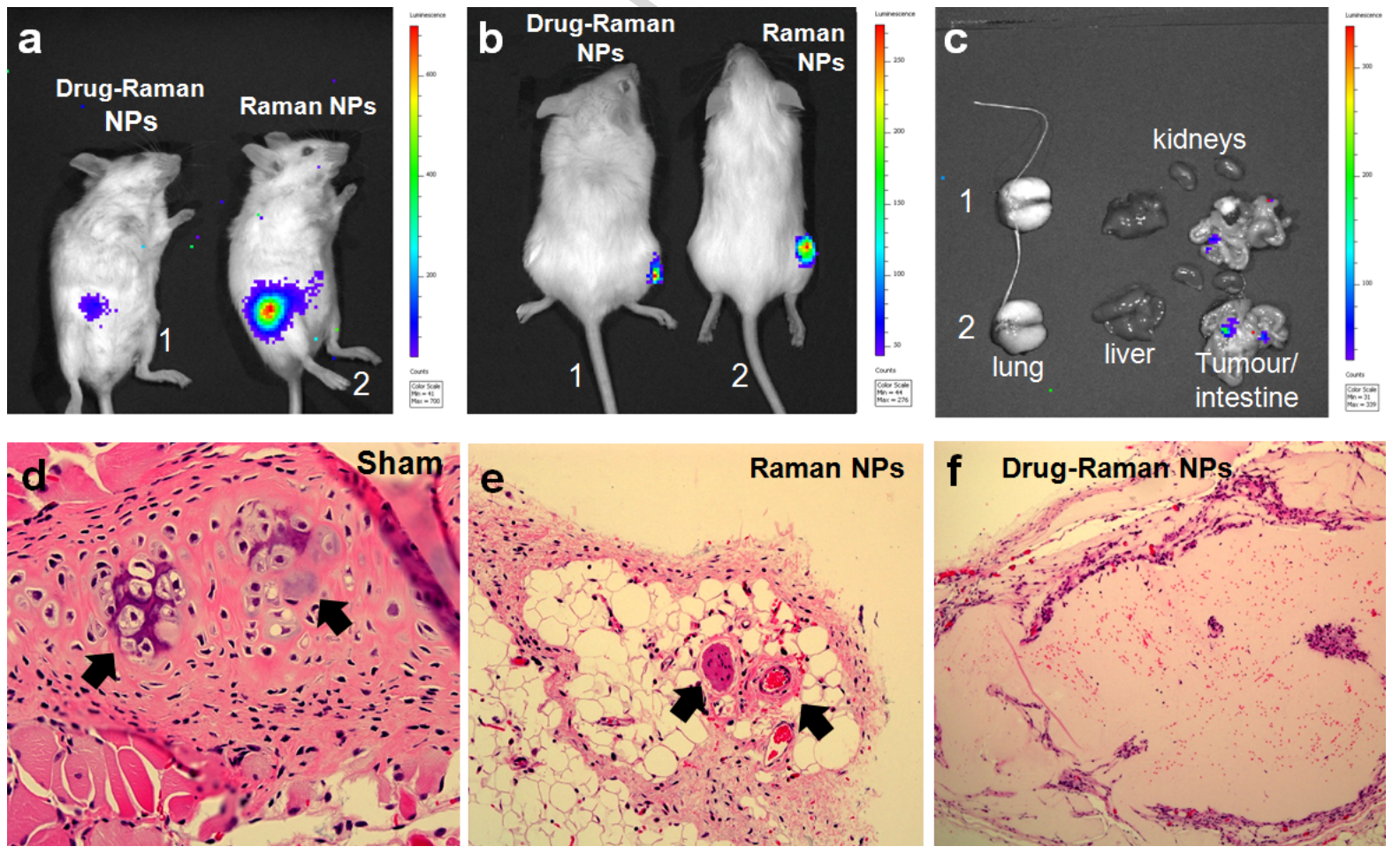

Figure 4. Tumour size assessment after nanoparticle in vivo administration. Tomography live imaging of mice injected treated with $1.78 \times 10^{9}$ NPs of Drug-Raman NPs and Raman NPs (without antibody-drug conjugate). Representative imaging of individual mice from each treated group ( $\mathrm{n}=10$ animals) is shown, with the same scale of photon flux indicating luciferase activity $(\mathbf{A}, \mathbf{B})$. (C) Evaluation of luciferase luminescence in mice whole body organs. Hematoxylin and eosin (H\&E) stains of (D) sham treated group (mice tissue without nanoparticle's treatment), (E) Raman NPs and (F) Drug-Raman NPs treated groups, in mice bearing xenografts tumours. Several tumour clones are noted in sham and Raman NPs treated groups (arrows). In D-F the blue dots indicate the cell nuclei. 
In order to test nanoparticle uptake and biodistribution in whole body mice and predominantly in tumour tissue, tomography imaging measuring epifluorescence was carried out in the same animals as in Figure 4A-C. Representative tomographic images of individual mice (Figure 5A,B) and whole body organs (Figure 5C) from each treated group are shown, with the same scale of epi-fluorescence indicating emission signal from the Raman reporter, DTTC $($ Emission $=780-800 \mathrm{~nm})$. Drug-Raman NPs actively accumulates in xenograft tumour (5fold when compared to Raman NPs only), measured by increased emission signal from Raman reporter (Figure 5B). Interestingly, Raman NPs also accumulates in tumour but with a very low fluorescence signal $( \pm 10-15 \%)$. This may occur due to passive accumulation of the nanoparticles via the enhanced permeability and retention (EPR) effect. Tumours present defective vasculature and poor lymphatic drainage due to their rapid growth and, therefore, nanoparticles tend to extravasate the vasculature and accumulate preferably at the tumour site.[27;28] Tomography data further indicate that nanoparticles were gradually accumulated in tumour site and that most of the accumulated particles stayed in the tumour for 72 hours. Tumour specificity of Drug-Raman NPs is proved by measuring the epi-fluorescence emission signal from the Raman reporter in the excised organs from mice (Figure 5C). As shown, only Drug-Raman NP treated group produced intense regions of fluorescence relative to control (Raman NP), particularly in tumour boundaries where blood vessels are well perfused. These results clearly indicate that the Drug-Raman NP were able to specifically detect EGFR-positive tumours in vivo.

Seventy-two hours after the tail-vein injection, the SERS spectra of the tumour site were measured through the tumour tissues excised from the mice. The signal of the tumour site perfectly resembled the SERS spectra of the pure Drug-Raman NPs (see Figure 2D), whereas a very weak SERS signal was detected from Raman NPs only (without antibody-drug 
conjugate) or other anatomical locations (i.e., the opposite leg) (Figure 5D). The SERS

measurements were carried out at the three locations to confirm the accumulation, which was in accord with the epi-fluorescence imaging (Figure 5B). The in vivo SERS spectra were identical to that obtained in vitro, however the absolute intensities of the characteristic SERS peak at $508 \mathrm{~cm}^{-1}$ for Drug-Raman NPs were higher ( 4.5-fold), when compared to RamanNPs only. These findings assured us of the value of our gold nanoantennas as SERS reporters for in vivo applications.
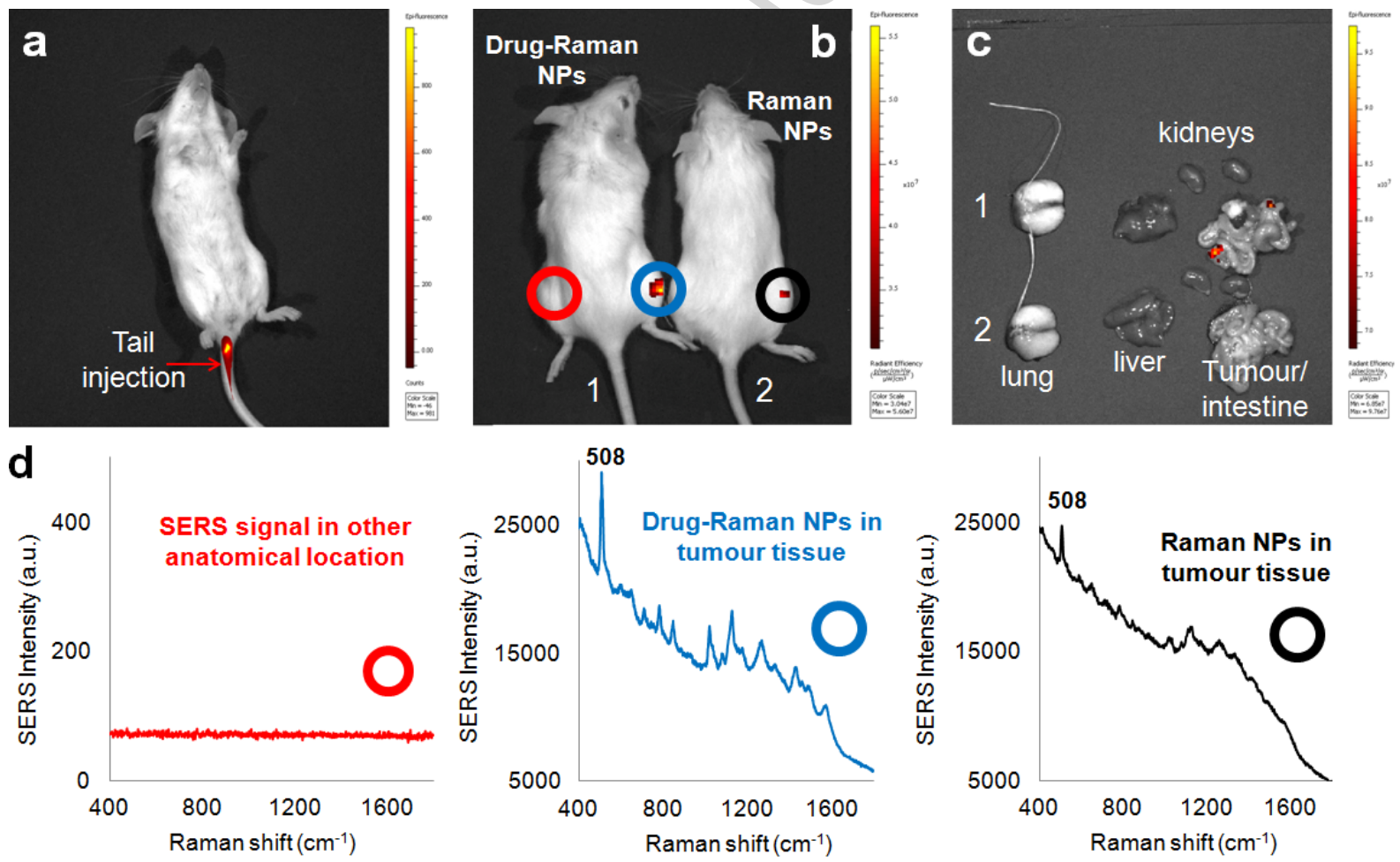

Figure 5. Drug-Raman NPs uptake and biodistribution in whole body mice. Representative imaging of individual mice from each treated group $(n=10$ animals $)$ is shown, with the same scale of epi-fluorescence $(\mathbf{A}, \mathbf{B})$ indicating emission signal from the Raman reporter, DTTC (Emission $=780-800 \mathrm{~nm})$. (C) Evaluation of nanoparticles' distribution in mice organs treated with Raman- and Drug-Raman NPs. (D) SERS spectra of the tumour site, measured through the tumour tissues excised from the mice, of Drug-Raman NPs (blue line), Raman NPs only (black line) and other anatomical locations (i.e., the opposite leg, red line). Characteristic SERS peak at $508 \mathrm{~cm}^{-1}$. 


\section{CONCLUSIONS}

In summary, we prepared $\sim 90 \mathrm{~nm}$ AuNPs surrounded by an active Raman reporter encapsulated in a polymer and an antibody-drug conjugate, which exhibited strong SERS intensities. These nanoconjugates are a highly sensitive SERS reporter nanoformulation with excellent signal stability, both in vitro and in vivo. Results confirmed that the SERS signatures obtained in vivo matched those collected from drug/Raman coded AuNPs in vitro and those from bulk solution. Moreover, we highlight the immense in vivo potential for drug/Raman coded nanoantennas. These cetuximab-conjugated drug/Raman-NPs were able to target and inhibit tumour progression, leading to tumour size decrease, while simultaneously allowing for spectroscopic detection of relatively small xenograft tumours. The high sensitivity and tumour specificity of cetuximab-conjugated Raman-NPs confirms their exceptional potential as non-toxic nanotheranostic procedure. Our finding pave the way for new concepts, that nanotherapy and nanodiagnostics can offer to cancer research via engineered fine-tuned drug/SERS gold nanoantennas that can be used for clinical or pre-clinical in vivo imaging and tumour suppression. In comparison to other Raman detection methods, our approach offers both greater sensitivity (signal strength) and excellent specificity to inhibit tumour cells in an in vivo mice model.

\section{MATERIALS AND METHODS}

\section{Synthesis of drug/Raman coded AuNPs}

Spherical AuNPs with $~ 90 \mathrm{~nm}$ were prepared using a seeded growth approach as described elsewhere.[29] Then, AuNPs were incubated with freshly prepared DTTC reporter (3,3'Diethylthiatricarbocyaniniodid, Sigma) solution $(4 \mu \mathrm{M})$ by adding it dropwise to a mixture of $1.56 \times 10^{11} \mathrm{NPs}$ and $0.2 \%(\mathrm{w} / \mathrm{v})$ of sodium dodecyl sulfate (SDS, Sigma). After $20 \mathrm{~min}$, a $\alpha$ Mercapto- $\omega$-carboxy PEG solution (HS-C ${ }_{2} \mathrm{H}_{4}-\mathrm{CONH}-\mathrm{PEG}-\mathrm{O}-\mathrm{C}_{3} \mathrm{H}_{6}-\mathrm{COOH}, \mathrm{MW} .5000 \mathrm{Da}$, 
Sigma) was added in 10 -fold excess $(2.25 \mathrm{mg} / \mathrm{mL})$ dropwise to the Raman-AuNPs. Excess PEG was removed by centrifugation $\left(21.460 \times \mathrm{g}, 30 \mathrm{~min}, 4^{\circ} \mathrm{C}\right)$. Cetuximab (Erbitux ${ }^{\circledR}$, Merck) was bound to the SERS-NPs by a carbodiimide chemistry assisted by Nhydroxisuccinimide using an EDC/NHS coupling reaction between the carboxylated PEG spacer and the amine terminal group of the antibody. Briefly, $1.56 \times 10^{11}$ NPs-DTTC-PEG, $1.24 \mathrm{mg} / \mathrm{mL}$ N-hydroxysulfosuccinimide (sulfo-NHS, Sigma) and $312 \mu \mathrm{g} / \mathrm{mL}$ EDC (1-Ethyl3-(3-dimethylaminopropyl)carbodiimide, Sigma) were incubated in $10 \mathrm{mM}$ MES (2-(Nmorpholino)ethanesulfonic acid, Sigma) at pH 6.1 and allowed to react for 30 minutes to activate the carboxylic groups. After this, activated AuNPs were washed once with $10 \mathrm{mM}$ MES, pH 6.1 and used immediately. Cetuximab antibody were added to the mixture at a final concentration of $45 \mu \mathrm{g} / \mathrm{mL}$ and allowed to react for 16 hours at $25^{\circ} \mathrm{C}$. The NPs-DTTC-PEGAntibody (drug/Raman coded AuNPs) were centrifuged at $21.460 \times \mathrm{g}$ for $30 \mathrm{~min}$ at $4^{\circ} \mathrm{C}$ and washed three times with Mili-Q water.

\section{Physicochemical characterization of drug/Raman coded AuNPs}

All AuNPs and Raman reporter absorbance spectra were acquired in a Cary Eclipse (Varian) using an Ultra-Micro quartz cell (Hëllma). Raman spectra were acquired using a Jobin Yvon micro-Raman spectroscope with a 300mW 785nm diode laser as source. Spectral data was collected using a $10 \times$ microscope objective over the range $400-1800 \mathrm{~cm}^{-1}$ with a $10 \mathrm{sec}$ integration time. For nanoparticle size determination, samples were analyzed through Dynamic Light Scattering (DLS) with a nanoparticle analyzer from Horiba Scientific Nano Portica sz-100 at an angle of $90^{\circ} \mathrm{C}$ and at $25^{\circ} \mathrm{C}$, in water. Zeta potential measurements of all AuNP-conjugates were made using a Zeta PALS (Brookhaven) analyzer at $25^{\circ} \mathrm{C}$, in water and $\mathrm{KCl}$ 0.1 M. Dark field light scattering microscopy was performed on a Zeiss Axioplan 2 Imaging Microscope with a HAL 100 illuminator usually used as light source for transmittedlight and reflected-light techniques, and images acquired using a Nikon DXM1200F digital 
camera. Dark-field images of AuNPs were acquired by mounting the cover slips with AuNPs mounted with $10 \mu 1$ of glycerol and a cover glass.

\section{In vitro incubation of drug/Raman coded AuNPs}

HT-adenocarcinoma cells were grown in Dulbecco's modified Eagle's medium with Glutamax (DMEM, Invitrogen), 10\% heat inactivated foetal bovine serum (Invitrogen), $100 \mathrm{U} / \mathrm{ml}$ penicillin and $100 \mu \mathrm{g} / \mathrm{mL}$ streptomycin (Invitrogen), and maintained at $37^{\circ} \mathrm{C}$ in $5 \% \mathrm{CO}_{2}$. Cells were seeded in 24-well plates at a density of $1 \times 10^{5}$ cells/well and grown for 24 hours prior to incubation with AuNPs. On the day of incubation, $1.78 \times 10^{9} \mathrm{NPs}$ of drug/Raman coded AuNPs were added to cells at approximately 50\% confluence in DMEM supplemented only with $10 \%$ heat inactivated fetal bovine serum. After 24 hours, cells were fixed with $-20^{\circ} \mathrm{C}$ methanol (anhydrous $99.8 \%$, Sigma) for $10 \mathrm{~min}$ and with $-20^{\circ} \mathrm{C}$ acetone (for $\mathrm{HPLC} \geq 99.9 \%$, Sigma) for 10 min on cover slips. The cover slips were washed twice in PBS. After DAPI staining, one drop of aqueous mounting medium was added on the cover slip and inverted carefully on a glass slide. The images were acquired in a Zeiss confocal microscope.

\section{Cytotoxicity evaluation}

Standard MTT [3-(4,5-dimethylthiazol-2-yl)-2,5-diphenyltetrazoliumbromide] reduction assay (Invitrogen) was performed to determine cytotoxicity following transfection with Raman-NPs and drug/Raman-NPs as described before.[30]. After 24 hours, NPs $\left(1.78 \times 10^{9}\right.$ NPs) were added to the wells and cells further incubated for 24, 48 and 72 hours. Following medium removal, cells were washed twice with sterile $1 \times$ PBS and incubated for 2 hours with $300 \mu 1$ of fresh medium with serum supplemented with $16.7 \mu 1$ of sterile MTT stock solution ( $5 \mathrm{mg} / \mathrm{mL}$ in $1 \times$ PBS). Next, medium was removed, formazan crystals resuspended in $300 \mu 1$ of dimethyl sulfoxide (Sigma), and absorbance measured at $540 \mathrm{~nm}$ as a working wavelength and $630 \mathrm{~nm}$ as reference using a Microplate reader Infinite M200 with Absorbance module 
(Tecan). Cell viability was normalized to that of cells cultured in the culture medium with PBS treatment.

In vivo targeting of drug/Raman coded AuNPs

Prior to nanoparticle's injection, B6 albino mice were injected in the right leg with $\left(1 \times 10^{5}\right.$ cells) HT-29-luc-D6 Bioware® Cell Line (P/N 119264, PerkinElmer®). Mice were anesthetized by inhalation of isoflurane vaporized at concentrations of up to $4 \%$. Eight weeks after xenograft tumour induction $1.78 \times 10^{9}$ NPs of Raman and drug/Raman coded AuNPs were administered in mice. After 72 hours, mice live images were taken with the IVIS® imaging system (Lumina, PerkinElmer) from mice bearing tumours from HT-29-luc-D6 Bioware ${ }^{\circledR}$ cells $\left(\mathrm{n}=10\right.$ animals per treated group). The IVIS ${ }^{\circledR}$ in vivo imaging system uses an optical imaging technology to facilitate non-invasive longitudinal monitoring of tumour progression and nanoparticles' cell trafficking in living mice, across the blue to NIR wavelength region. IVIS $®$ images were taken under excitation filter of $690 \mathrm{~nm}$ and emission filter of $790 \mathrm{~nm}$. After live imaging, mice organs were dissected and prepared for Haematoxylin and Eosin staining (H\&E).

\section{Acknowledgements}

JC acknowledges FCT grant (SFRH/BD/62957/2009). PVB thanks CIGMH/FCT/MCES (PEst-OE/SAU/UI0009/2011). 


\section{REFERENCES}

[1] A.J. Wilson, K.A.Willets, Surface-enhanced Raman scattering imaging using noble metal nanoparticles. Wiley Interdisciplinary Reviews-Nanomedicine and Nanobiotechnology 5 (2013)180-189.

[2] P.L. Stiles, J.A. Dieringer, N.C. Shah, R.R. Van Duyne, Surface-Enhanced Raman Spectroscopy. Annual Review of Analytical Chemistry 1 (2008) 601-626.

[3] J. Ando, T. Yano, K. Fujita, S. Kawata, Metal nanoparticles for nano-imaging and nano-analysis. Physical Chemistry Chemical Physics 15 (2013) 13713-13722.

[4] J. Conde, J. Rosa, J.C. Lima, P.V. Baptista, Nanophotonics for Molecular Diagnostics and Therapy Applications. International Journal of Photoenergy 2012 (2012) 1-12.

[5] X. Wang, X. Qian, J.J. Beitler, Z.G. Chen, F.R. Khuri, M.M. Lewis, H.J. Shin, S. Nie, D.M .Shin, Detection of circulating tumor cells in human peripheral blood using surface-enhanced Raman scattering nanoparticles. Cancer Res. 71 (2011) 1526-1532.

[6] R.A. Alvarez-Puebla, L.M. Liz-Marzan, SERS Detection of Small Inorganic Molecules and Ions. Angewandte Chemie-International Edition 51 (2012) 1121411223.

[7] P. Mi, D. Kokuryo, H. Cabral, M. Kumagai, T. Nomoto, I. Aoki, Y. Terada, A. Kishimura, N. Nishiyama, K. Kataoka, Hydrothermally synthesized PEGylated calcium phosphate nanoparticles incorporating Gd-DTPA for contrast enhanced MRI diagnosis of solid tumors. J Control Release 174C (2013) 63-71.

[8] M. Vendrell, K.K. Maiti, K. Dhaliwal, Y.T. Chang, Surface-enhanced Raman scattering in cancer detection and imaging. Trends in Biotechnology 31 (2013) 249257.

[9] R. Jin, Nanoparticle clusters light up in SERS. Angew. Chem. Int. Ed Engl. 49, 28262829 (2010). 
[10] X.M. Qian, X. Zhou, S.M. Nie, Surface-Enhanced Raman Nanoparticle Beacons Based on Bioconjugated Gold Nanocrystals and Long Range Plasmonic Coupling. Journal of the American Chemical Society 130 (2008) 14934-14935.

[11] I.H. El-Sayed, X.H. Huang, M.A. El-Sayed, Surface plasmon resonance scattering and absorption of anti-EGFR antibody conjugated gold nanoparticles in cancer diagnostics: Applications in oral cancer. Nano Letters 5 (2005) 829-834.

[12] K.V. Kong, Z. Lam, W.D. Goh, W.K. Leong, M. Olivo, Metal Carbonyl-Gold nanoparticle conjugates for live-cell SERS imaging. Angewandte ChemieInternational Edition 51 (2012) 9796-9799.

[13] J.C. Kah, K.W. Kho, C.G. Lee, C. James, R. Sheppard, Z.X. Shen, K.C. Soo, M.C. Olivo, Early diagnosis of oral cancer based on the surface plasmon resonance of gold nanoparticles. Int. J. Nanomedicine. 2 (2007) 785-798.

[14] X.M. Qian, X.H. Peng, D.O. Ansari, Q. Yin-Goen, G.Z. Chen, D.M. Shin, L. Yang, A.N. Young, M.D. Wang, S. Nie, In vivo tumor targeting and spectroscopic detection with surface-enhanced Raman nanoparticle tags. Nature Biotechnology 26 (2008) 8390.

[15] C. Bokemeyer, E. Van Cutsem, P. Rougier, F. Ciardiello, S. Heeger, M. Schlichting, I. Celik, C.H. Köhne, Addition of cetuximab to chemotherapy as first-line treatment for KRAS wild-type metastatic colorectal cancer: Pooled analysis of the CRYSTAL and OPUS randomised clinical trials. European Journal of Cancer 48 (2012)1466-1475.

[16] M.V. Yigit, L. Zhu, M.A. Ifediba, Y. Zhang, K. Carr, A. Moore, Z. Medarova, Noninvasive MRI-SERS imaging in living mice using an innately bimodal nanomaterial. ACS Nano. 5 (2011) 1056-1066.

[17] G.F. Paciotti, L. Myer, D. Weinreich, D. Goia, N. Pavel, R.E. McLaughlin, L. Tamarkin, Colloidal gold: A novel nanoparticle vector for tumor directed drug delivery. Drug Delivery 11 (2004) 169-183. 
[18] J. Conde, A. Ambrosone, V. Sanz, Y. Hernandez, V. Marchesano, F. Tian, H. Child, C.C. Berry, Ibarra MR, Baptista PV, C. Tortiglione, J.M.de la Fuente, Design of multifunctional gold nanoparticles for in vitro and in vivo gene silencing. ACS Nano 6 (2012) 8316-8324.

[19] J. Conde, F. Tian, Y. Hernández, C. Bao, D. Cui, K.P. Janssen, M.R. Ibarra, P.V. Baptista, T. Stoeger, J.M. de la Fuente, In vivo tumor targeting via nanoparticlemediated therapeutic siRNA coupled to inflammatory response in lung cancer mouse models. Biomaterials 34 (2013) 7744-7753.

[20] J. Conde, P.V. Baptista, Y. Hernandez, V. Sanz, J.M. de la Fuente, Modification of plasmid DNA topology by 'histone-mimetic' gold nanoparticles. Nanomedicine 7 (2012) 1657-1666.

[21] J.T. Krug, G.D. Wang, S. Emory, S.M. Nie, Efficient Raman enhancement and intermittent light emission observed in single gold nanocrystals. Journal of the American Chemical Society 121 (1999) 9208-9214.

[22] U. Mahmood, R. Weissleder, Near-infrared optical imaging of proteases in cancer. Molecular Cancer Therapeutics 2 (2003) 489-496.

[23] J.N. Anker, W.P. Hall, O. Lyandres, N.C. Shah, J. Zhao, R.P. Van Duyne, Biosensing with plasmonic nanosensors. Nat. Mater. 7 (2008) 442-453.

[24] J. P. Spano, R. Fagard, J.C. Soria, O. Rixe, D. Khayat, G. Milano, Epidermal growth factor receptor signaling in colorectal cancer: preclinical data and therapeutic perspectives. Annals of Oncology 16 (2005) 189-194.

[25] H. Shi, X.X. He, Y. Yuan, K.M. Wang, D. Liu, Nanoparticle-Based Biocompatible and Long-Life Marker for Lysosome Labeling and Tracking. Analytical Chemistry 82 (2010) 2213-2220.

[26] C.M.J. Hu, L.F. Zhang, Therapeutic Nanoparticles to Combat Cancer Drug Resistance. Current Drug Metabolism 10 (2009) 836-841. 
[27] J. Conde, G. Doria, P. Baptista, Noble metal nanoparticles applications in cancer. J. Drug Deliv. 2012, (2012) 751075.

[28] P.R. Gil, W.J. Parak, Composite nanoparticles take aim at cancer. ACS Nano. 2 (2008) 2200-2205.

[29] C. Ziegler, A. Eychmüller, Seeded Growth Synthesis of Uniform Gold Nanoparticles with Diameters of 15-300 nm. J. Phys. Chem. C. 115 (2011) 4502-4506.

[30] J. Conde, M. Larguinho, A. Cordeiro, L.R. Raposo, P.M. Costa, S. Santos, M.S. Diniz, A.R. Fernandes, P.V. Baptista, Gold-nanobeacons for gene therapy: evaluation of genotoxicity, cell toxicity and proteome profiling analysis. Nanotoxicology 8 (2014) 521-532. 


\section{FIGURES}
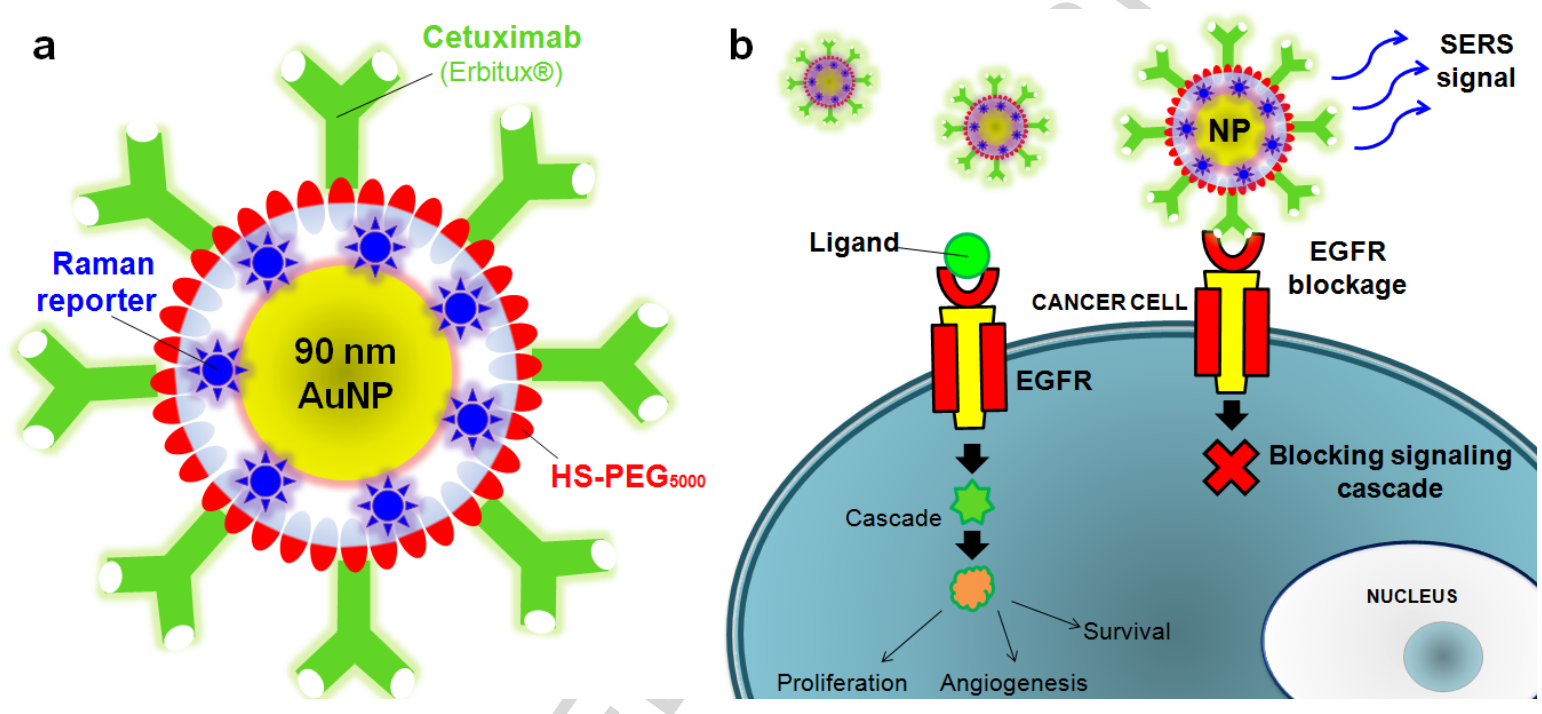

Figure 1. (A) Antibody-Drug gold SERS nanoantennas - 90nm AuNPs surrounded by a Raman reporter, encapsulated and entrapped by a larger polyethylene glycol (PEG) polymer and covered with a FDA approved antibody-drug conjugate, the Cetuximab (Erbitux $\left.{ }^{\circledR}\right)$. (B) The Drug-Raman NPs can easily bind to EGF receptors (EGFR), blocking the EGF protein from reaching the cancer cells and inhibit the signalling cascade. The activation of this cascade will consequently stop proliferation and survival of targeted cells. 

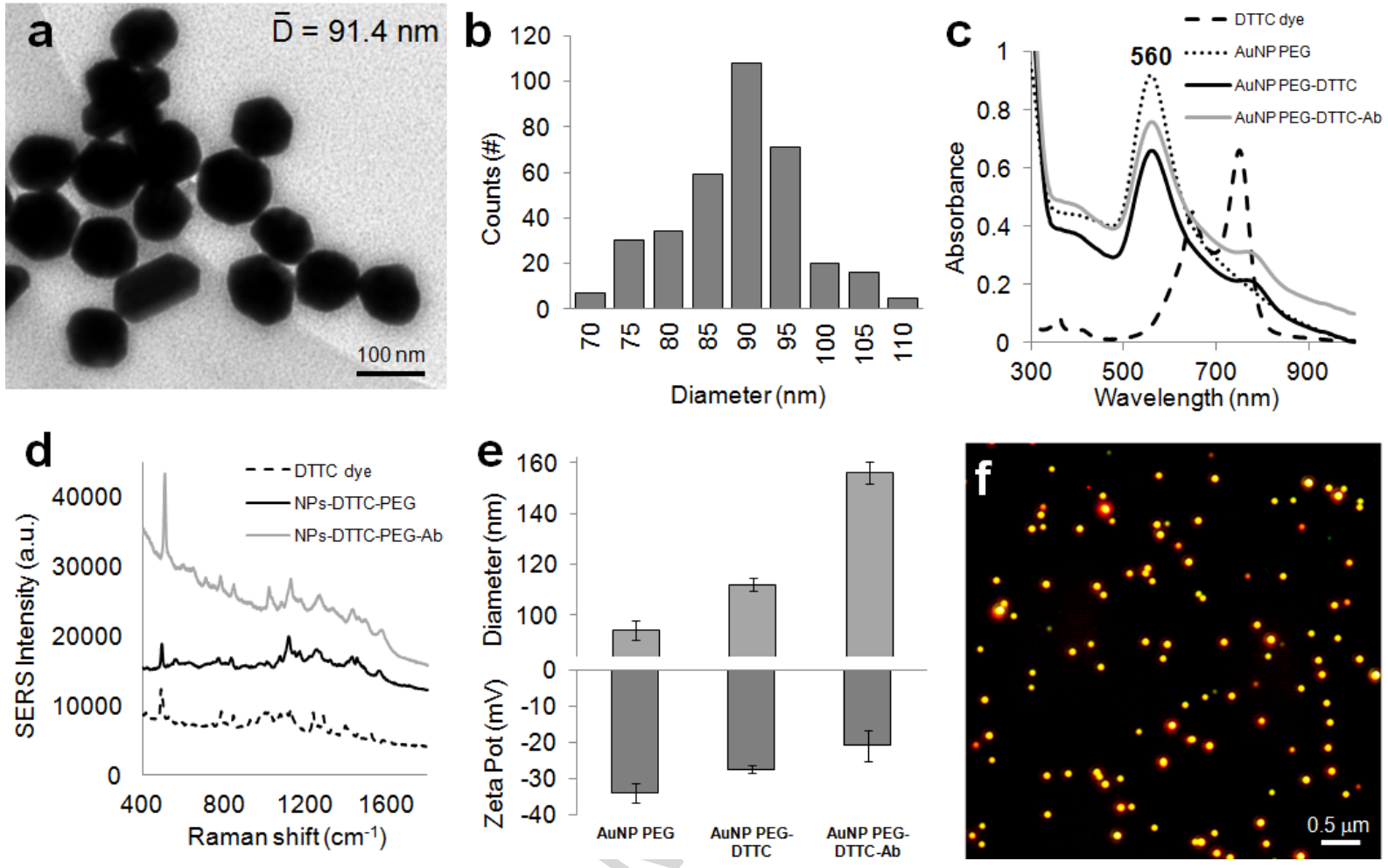

Figure 2. Physicochemical characterization of tested AuNPs (A) Transmission electron microscopy images of spherical naked AuNPs (scale bar, $100 \mathrm{~nm}$ ) with an average diameter of $91.4 \pm 8.9 \mathrm{~nm}(\mathbf{B})$. (C) Optical extinction spectra with an SPR band centred at $560 \mathrm{~nm}$. Also shown is a DTTC absorbance spectrum with characteristic peaks at 650 and $750 \mathrm{~nm}$ (D) Raman spectra of AuNP-conjugates with a characteristic SERS peak at $508 \mathrm{~cm}^{-1}$. (E) Hydrodynamic light scattering (DLS) in water and zeta-potential data of all AuNP-conjugates. (F) Dark-field light-scattering microscopy of Drug-Raman NPs, i.e. NPs-PEG-DTTC-Ab (scale bar, $0.5 \mu \mathrm{m}$ ). 

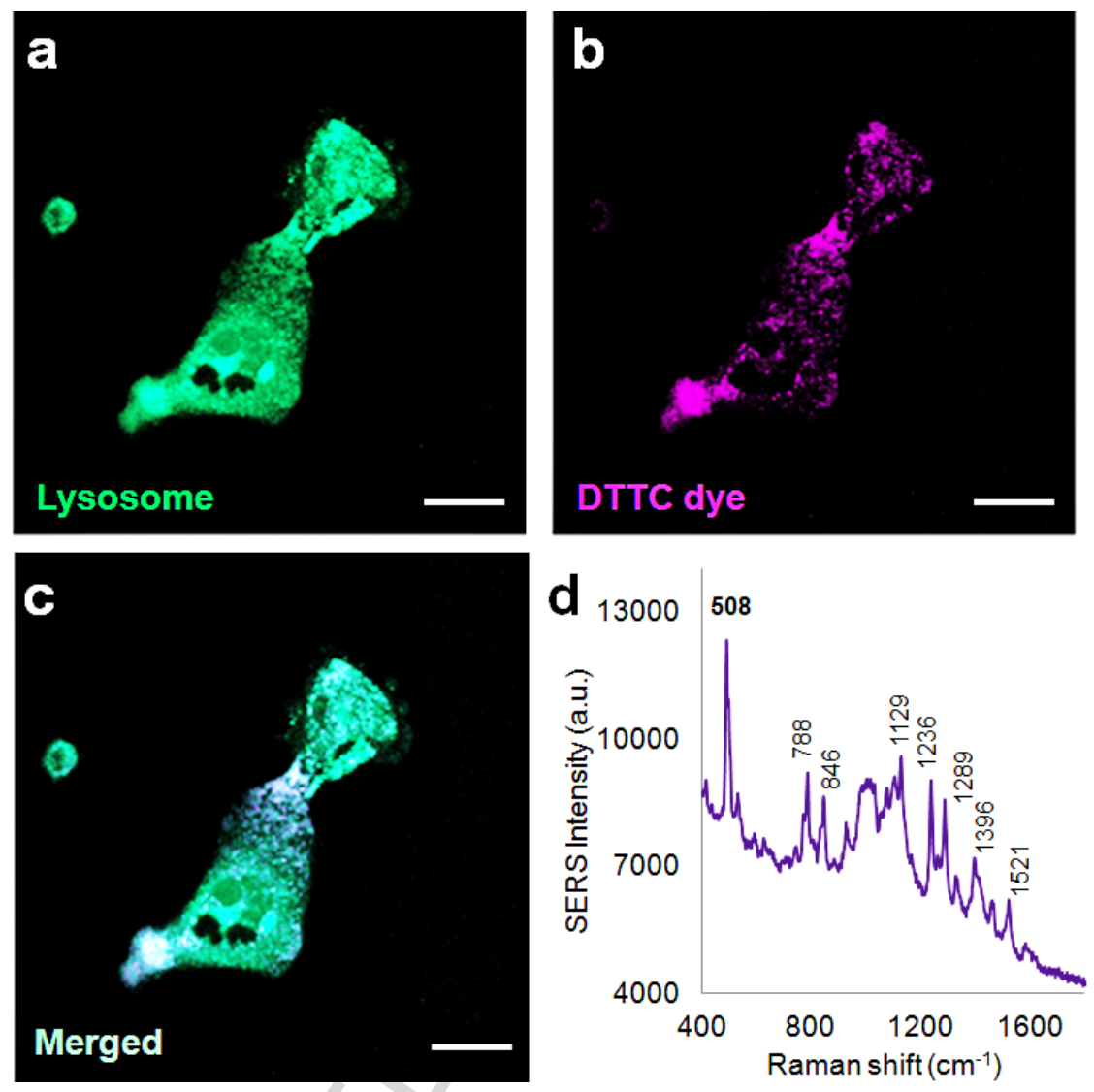

Figure 3. Representative confocal images of HT-adenocarcinoma cells incubated for 24 hours with Drug-Raman NPs $\left(1.78 \times 10^{9}\right.$ NPs). Shown is lysosome signal via LysoTracker Green (A), Raman reporter (DTTC dye) emission signal (B) and co-localization signal between DrugRaman NPs and lysosomes $(\mathbf{C})$ (scale bars, $10 \mu \mathrm{m}$ ). (D) Characteristic Raman spectra and peaks of Drug-Raman NPs in HT-adenocarcinoma cells, with a characteristic SERS peak at $508 \mathrm{~cm}^{-1}$. 

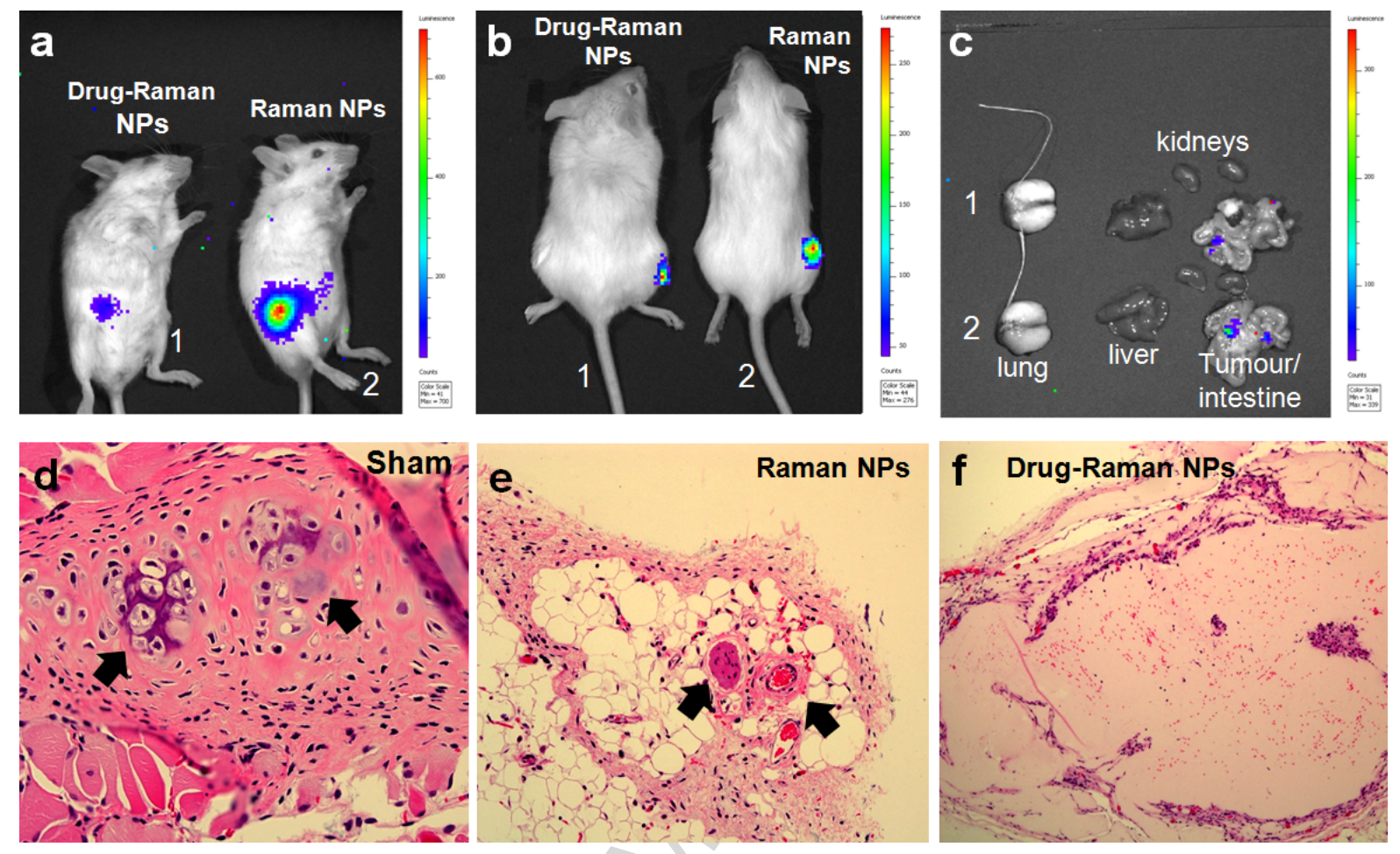

Figure 4. Tumour size assessment after nanoparticle in vivo administration. Tomography live imaging of mice injected treated with $1.78 \times 10^{9}$ NPs of Drug-Raman NPs and Raman NPs (without antibody-drug conjugate). Representative imaging of individual mice from each treated group ( $\mathrm{n}=10$ animals) is shown, with the same scale of photon flux indicating luciferase activity $(\mathbf{A}, \mathbf{B})$. (C) Evaluation of luciferase luminescence in mice whole body organs. Hematoxylin and eosin (H\&E) stains of (D) sham treated group (mice tissue without nanoparticle's treatment), (E) Raman NPs and (F) Drug-Raman NPs treated groups, in mice bearing xenografts tumours. Several tumour clones are noted in sham and Raman NPs treated groups (arrows). In D-F the blue dots indicate the cell nuclei. 

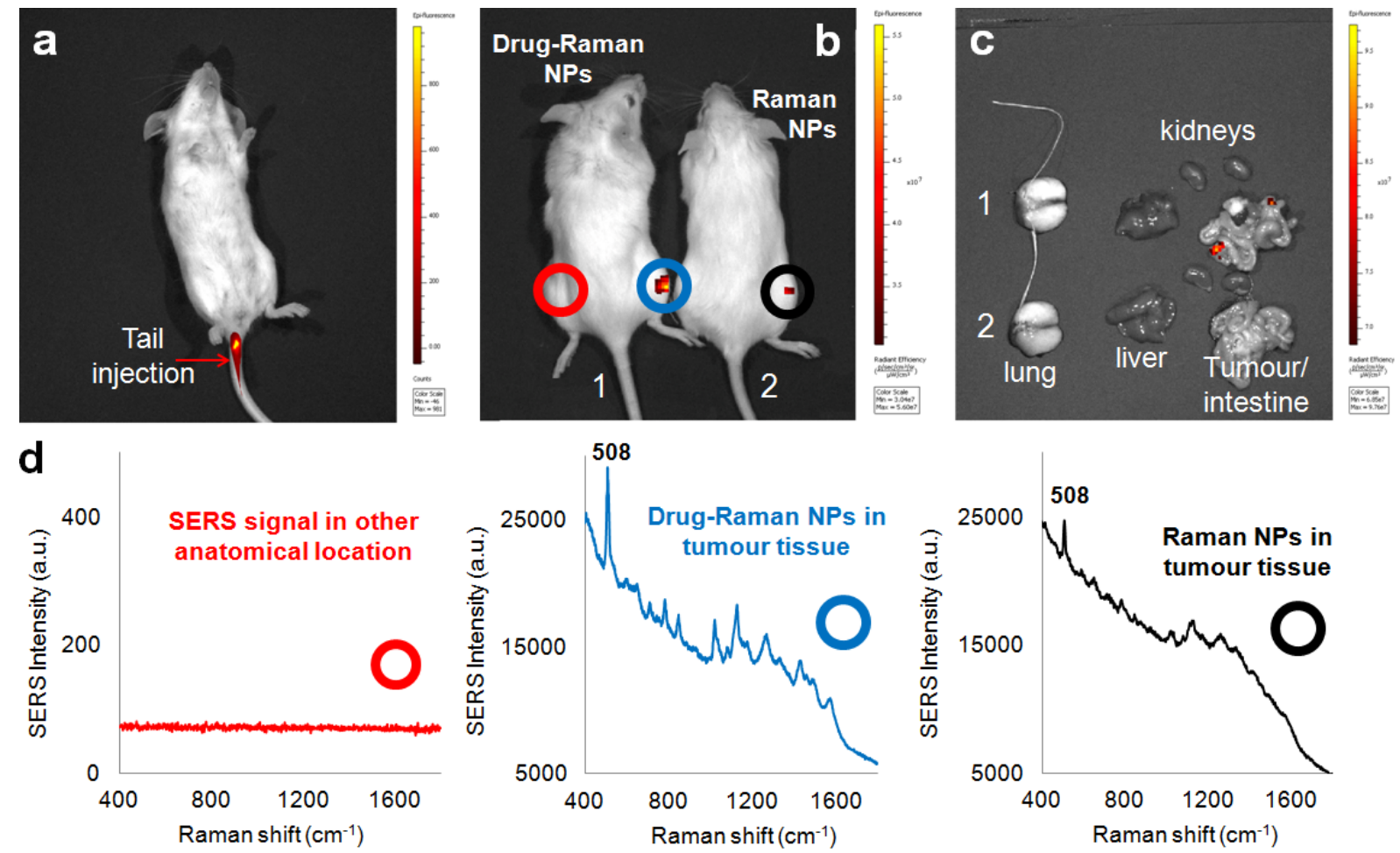

Figure 5. Drug-Raman NPs uptake and biodistribution in whole body mice. Representative imaging of individual mice from each treated group $(n=10$ animals $)$ is shown, with the same scale of epi-fluorescence (A,B) indicating emission signal from the Raman reporter, DTTC (Emission $=780-800 \mathrm{~nm}$ ). (C) Evaluation of nanoparticles' distribution in mice organs treated with Raman- and Drug-Raman NPs. (D) SERS spectra of the tumour site, measured through the tumour tissues excised from the mice, of Drug-Raman NPs (blue line), Raman NPs only (black line) and other anatomical locations (i.e., the opposite leg, red line). Characteristic SERS peak at $508 \mathrm{~cm}^{-1}$. 


\section{GRAPHICAL ABSTRACT}

Antibody-Drug gold nanoantennas to use as nanotheranostic tool for in vivo imaging/detection and tumour inhibition. These smart Drug-Raman NPs can easily bind to EGF receptors, blocking the EGF protein from reaching the cancer cells and inhibit the signalling cascade, consequently stop proliferation and survival of targeted cells.

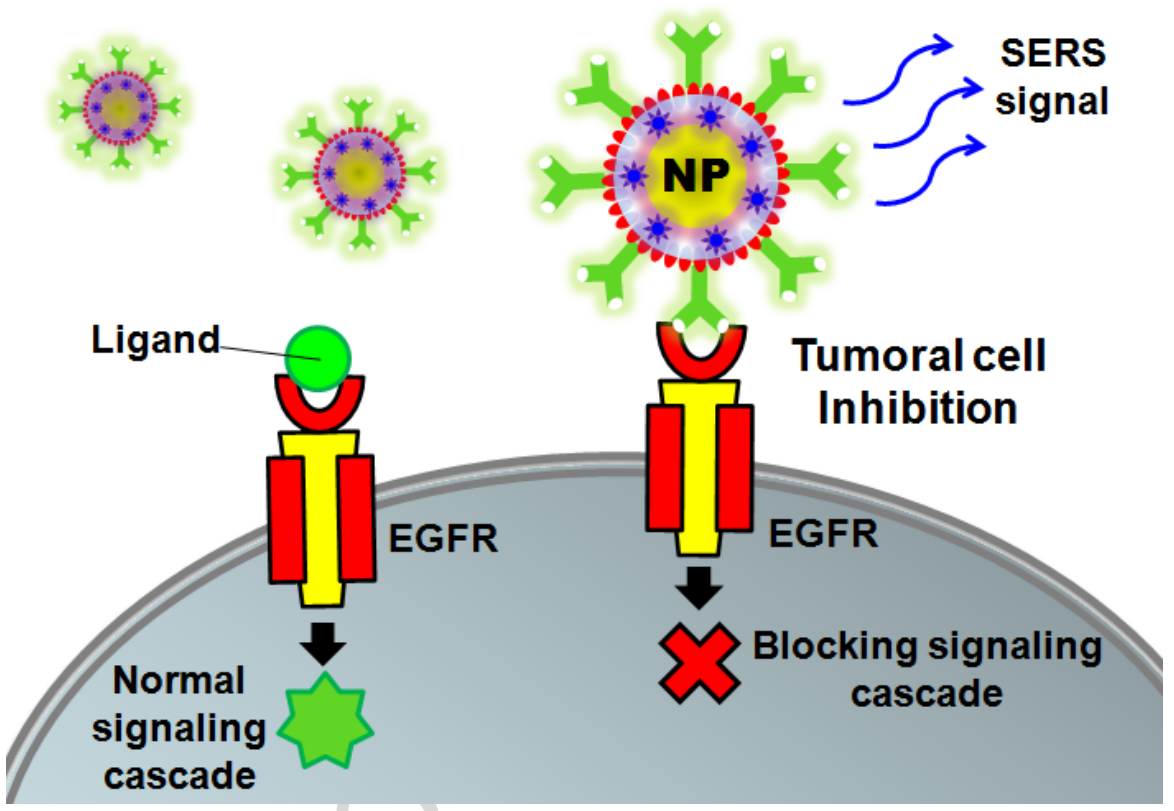

